\title{
The Impact of Cognitive Demands on Attention to Facial versus Situational Cues When Judging Emotions
}

\author{
Joann M. Montepare \\ RoseMary B. Fuss Center for Research on Aging and Intergenerational Studies, Lasell College, \\ Newton, Massachusetts, USA. \\ Email: jmontepare@lasell.edu \\ Received February 9 $9^{\text {th }}, 2011$; revised June $7^{\text {th }}, 2011$; accepted August 24 $4^{\text {th }}, 2011$.
}

\begin{abstract}
What information guides how perceivers evaluate people's emotional experiences? Some regard expressive facial cues to be the critical source of information whereas others argue that situational cues exert the decisive impact. This research explored an alternative view suggesting that both information sources are of consequence and that cognitive demands placed on perceivers play a pivotal role in determining what information they use. To test this view, perceivers were given discrepant combinations of facial and situational information about people's emotional experiences and identified what emotions they felt. Facial information influenced perceivers' judgments most often when demands were placed on their cognitive resources. In contrast, situational information had the greatest impact when cognitive demands were minimized. These findings shed light on the debate surrounding the issue of facial versus situational dominance and the process by which emotion judgments are made.
\end{abstract}

Keywords: Emotion Perception, Facial Cues, Cognitive Demand

\section{Introduction}

Since the publication of The Expression of the Emotions in Man and Animals (Darwin, 1872), researchers interested in nonverbal behavior have debated the nature of emotional expressions (DePaulo \& Friedman, 1998; Knapp \& Hall, 2007; Stanley \& Meyer, 2009). One question that has held center stage is the extent to which expressive facial cues are the critical source of information in emotion judgments. While some theorists regard facial information as preeminent, others contend that situational information drives judgments. The present research considers an alternative position that suggests that both sources of information play a role in emotion judgments and that basic cognitive mechanisms guide their relative impact.

Proponents of facial dominance view facial expressions as evolutionary adaptations that provide direct cues to basic affecttive states. Thus, when perceivers observe a person displaying an emotional expression, it is presumed that facial cues will dominate perceivers' judgments and override contradictory information provided by transient situational information (Buck, 1984; Ekman, 1992; Ekman \& Friesen, 1975; Tomkins, 19621963). However, it is acknowledged that from an early age people learn to conceal, control and manage their facial expressions so that in some situations emotion displays may be weak or ambiguous and contextual effects emerge (Izard, 1994; Matsumoto,Yoo, Hirayama, \& Petrova, 2005).

Early empirical evidence supporting facial dominance was provided by a procedure designed by Goodenough and Tinker (1931) in which perceivers were presented with discrepant combinations of facial information (pictures of a person's emotional facial expressions) and situational information (verbal descriptions of an emotional event a person encounters). When asked to identify what emotions the person experienced, research found that perceivers' judgments were strongly influenced by expressive facial cues (Ekman, Friesen, \& Ellsworth, 1982; Fernandez-Dols, Sierra, \& Ruiz-Belda, 1993; Fernandez-Dols, Wallbott, \& Sanchez, 1991; Nakamura, Buck, \& Kenny, 1990).
Several exceptions to the observed advantage of facial information have challenged the subordinate role of situational information. For instance, Fernandez-Dols, Wallbott and Sanchez (1991) found that when given an opportunity to categorize situations with emotion labels used for identifying facial expressions, perceivers made greater use of situational information in a Goodenough-Tinker task. Carroll and Russell (1996) provided the most theoretically compelling evidence for the impact of situational information. These researchers agree that faces evolved to provide socially adaptive information. However, facial expressions do not signal specific emotional states but rather reflect general affective dimensions of pleasantness and arousal. Thus, perceivers must look to situational information to determine the specific nature of a person's emotional experience. Moreover, situational information is especially critical to emotion judgments when facial and situational information vary along shared affective dimensions (see also Fridlund, 1994; Russell, 1997).

To demonstrate their position, Carroll and Russell (1996) told perceivers a story describing an emotional event and then showed them a picture of a person displaying a facial expression as in the Goodenough-Tinker procedure. The emotions that were represented shared similar valences with respect to pleasantness and arousal yet reflected emotions suggested to be basic and distinct (e.g., a story about a frightening event was paired with a picture depicting an angry expression). Perceivers identified what the person in the picture was feeling and their judgments coincided more with information provided by situational rather than facial information. Such findings are difficult to reconcile with the claim that facial expressions are preeminent in emotion judgments and signal specific emotional states. $^{1}$

${ }^{1}$ Ekman, Friesen and Ellsworth (1982) have suggested several methodologycal issues that could account for differences in the relative dominance of facial versus situational information in the emotion judgments that may be found when using procedures such as the Goodenough-Tinker (1931) one. An obvious factor, source clarity, were considered and eliminated as the basis for Carroll and Russell's (1996) findings. 
Can these opposing views be reconciled? Insights may be gained by moving beyond research designed to establish the relative dominance of information to investigating cognitive mechanisms that guide judgments when perceivers encounter diverse information. Advocates of both facial and situational dominance have acknowledged the need for research to explore the role cognitive mechanisms play in how perceivers process discrepant facial and situational information (Carroll \& Russell, 1996; Ekman, Friesen, \& Ellsworth, 1982; Fernandez-Dols, Wallbott, \& Sanchez, 1991). However, attention to this need has been unduly scant.

The present research rests on the assumption that perceivers routinely encounter emotion information from multiple sources in daily social exchanges and that general cognitive processes serve an important function in utilizing this information. Moreover, drawing on the social-cognitive conceptualization of person perception as a multi-level system (Gilbert \& Krull, 1988; Gilbert, Pelham, \& Krull, 1988), it is proposed that attention to facial information reflects a lower order judgment process that occurs automatically given the visual salience and communicative function of facial cues. In contrast, attention to situational information involves a higher order judgment process that requires more extensive cognitive effort given that contexts do not serve explicit communicative functions and involve multiple events. Given these distinctions, it is hypothesized that when perceivers encounter facial and situational information and attempt to make an emotion judgment, they will attend to facial information when their cognitive resources are strained. Such may have been the case in research that found evidence for facial dominance insofar as perceivers were typically asked to evaluate random combinations of information which is cognitively taxing. When cognitive demands are reduced, situational information will draw perceivers' attention. Such may have been the case in research that found evidence for situational dominance insofar as perceivers were given a greater opportunity to process situational information. To explore the merit of this cognitive-processing view, the present research replicated several experimental conditions utilized by Carroll and Russell (1996) and manipulated perceivers' level of cognitive demand as they attempted to make emotion judgments.

\section{Method}

\section{Participants}

Thirty-seven Caucasian psychology students (10 men and 27 women) between the ages of 17 and 23 (Mage = 19.32) volunteered to participate in the study. Approximately equal numbers were randomly assigned to one of two experimental conditions (i.e., low demand or high demand) and to one of two sets of stimulus faces.

\section{Stimulus Materials}

Two sets of faces were selected from those catalogued by Ekman and Friesen (1975). The first set consisted of 1 fearful male face, 1 angry female face, and 1 sad female face. The second set consisted of 1 sad female face, 1 fearful female face, and 1 angry male face. The presentation of faces in each set was randomly ordered. The faces were equated for size and reproduced in black and white ink for presentation on 8.5 x 11 in. overhead transparencies.

Three different stories describing emotional situations were selected from Carroll and Russell (1996) and are presented in Table 1 . The researchers developed (and tested) these stories to be unambiguous in emotional meaning. One story was written to suggest anger and was paired with the fearful faces. One story suggested fear and was paired with the angry faces. One story suggested disgust and was paired with the sad faces. The gender of the person in each story was modified appropriately to match the gender of the stimulus faces.

\section{Procedure}

Participants convened in small groups and were told that the study explored how people make judgments about what others are feeling. They were further told that they would be hearing about experiences of different people and seeing a picture of their face and that their task was to identify what each person was feeling. Participants were then presented with three face-story combinations (e.g., sad face-disgust situation; angry face-fear situation, fear face-anger situation).

The participants were read each story by a research assistant in a clear, non-emotional, uniform style who was blind to the experimental hypotheses. Immediately after the story was read, the corresponding face was presented on an overhead projector and participants were asked, "What emotion is the woman (man) feeling?” Participants responded by circling one of six emotions (happy, anger, sad, surprise, fear and disgust). The faces were kept in view for the same length of time as it took to read the stories to control for the potential confounding effects of differences in exposure to facial versus situational information.

\section{Experimental Manipulation}

Following the initial instructions, participants in the high

Table 1.

Stories depicting emotional situations.

\footnotetext{
This is a story of a man who had recently bought a new car. Today, he is walking back to his car across the parking lot after running errands at the post office. From the distance, he can see some kids around his car. Then he sees one of them holding one of the car's hubcaps. He yells at the kids and they take off to a nearby forest waving hubcaps in their hands. Now that he is close to his car, he can see that it is certainly missing its hubcaps.

Anger situation

This is a story about a woman who had never done anything really exciting in her life. One day she decided she had to do something

Fear situation exciting so she enrolled in a class for parachuting. Today is the day that she will make her first jump. She and her class are seated in the plane as it reaches the right altitude for parachute jumping. The instructor calls her name. It is her turn to jump. She refuses to leave her seat.

This is a story of a woman who went away on quite a long business trip. When she arrived home, even at the front door, she could smell something was wrong. As she entered the kitchen the smell grew even stronger. She found that she had forgotten to take out the

Disgust situation kitchen garbage. The rancid smell whooshed out as she closed the bag. The bag was so full that as she carried it to the curb it tore slightly and she could feel liquid from the bottom of the bag drip down her leg.
} 
demand condition were given the following instructions suggested by Patterson and Stockbridge (1998) before the facestory combinations were presented:

Now, in real life situations, where we make judgments about other people, we also have to attend to a variety of other concerns. To approximate these circumstances more closely, you will also be given a memory task - a grocery list to remember and recall after you answer what you think the person is feeling. Simply try to remember as much of the list as you can without writing down the items. After you describe what you think the person is feeling, you'll be asked to recall and write down as many of the items as you can.

Three different grocery lists were generated to go with each of the face-story combinations. An example of a grocery list is: a package of hot dog rolls, two bars of soap, graham crackers, and paper napkins. Grocery lists were read in the same manner as the stories. Each list was read twice before the story was read and the corresponding face was presented. After participants made their emotion judgment, they wrote down the grocery items they recalled. Participants in the low demand condition were not given any additional instructions. They were merely asked to view the faces, listen to the stories, and make their judgments. Following the experimental task, specific hypotheses were described, questions were addressed, and participants were thanked for their time. No indication was given that participants were aware of the issues being explored.

\section{Results}

As in Carroll and Russell's (1996) research, inspection of the percentage of participants selecting specific emotion responses were used to assess the impact of facial versus situational information on emotion judgments. Percentages within the low and high demand conditions are presented in Table 1 along with relevant percentages reported by Carroll and Russell (1996). According to predictions, judgments in the low demand condition should parallel those obtained when Carroll and Russell presented perceivers with facial information that conflicted with situational information. Percentages obtained in the high demand condition should parallel those observed when Carroll and Russell perceivers were provided only facial information.

\section{Low Cognitive Demand}

In the low cognitive demand condition, perceivers' judgments corresponded most closely with situational information. Perceivers most often identified people as feeling disgust when presented with sad faces and disgust situations. Similarly, they identified people most often as feeling anger when presented with fear faces and anger situations. They identified people most often as feeling fear when presented with angry faces and fear situations. Thus, perceivers attended more to situational than facial information when fewer demands were placed on their cognitive resources. This pattern paralleled what Carroll and Russell (1996) found when perceivers made emotion judgments when given contradictory facial and situational information

\section{High Cognitive Demand}

With greater cognitive demand, perceivers' judgments coincided more with facial information. Perceivers identified people most often as feeling sad when presented with sad faces and disgust situations, as feeling fear when presented with fear face and anger situations, and as feeling anger when presented with angry faces and fear situations. This pattern was similar to what Carroll and Russell (1996) found when perceivers judged emotions from facial information alone.

\section{Additional Analyses and Observations}

The systematic impact of cognitive demand was also evident from the frequency patterns shown in Figures 1-3. When re-
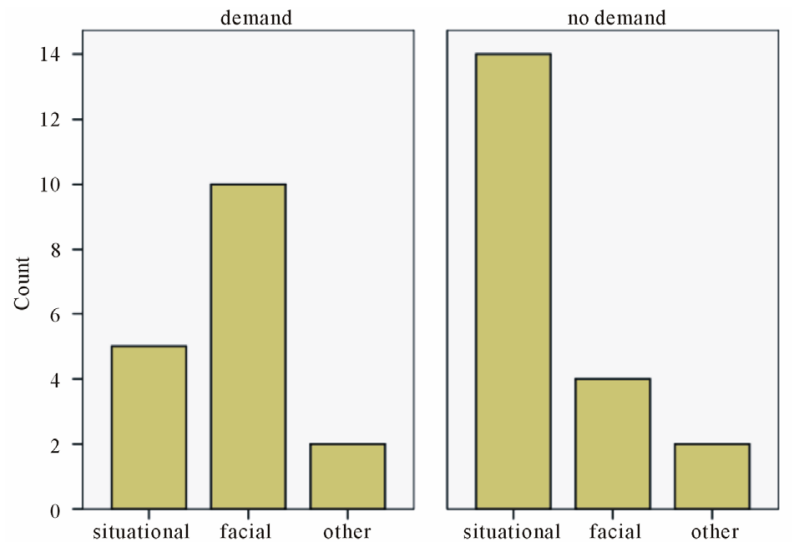

Figure 1.

Responses to facial, situational, and other information under low and high demand in the sad face-disgust situation condition.
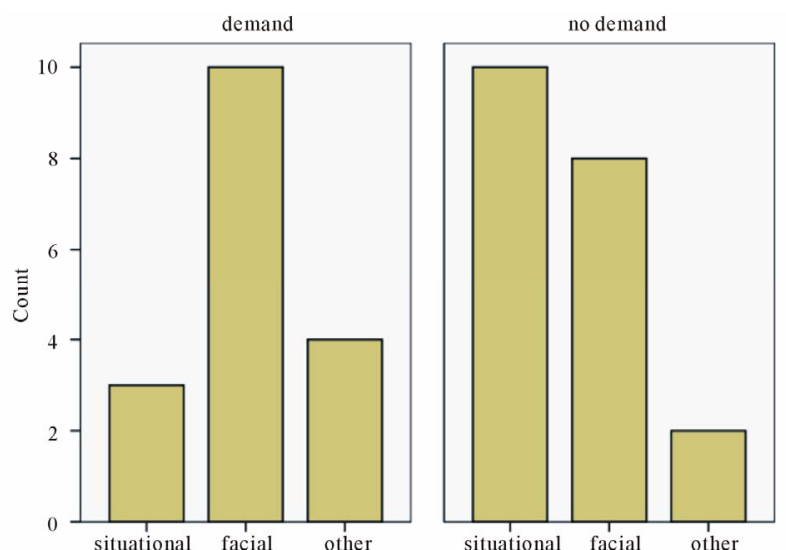

Figure 2.

Responses to facial, situational, and other information under low and high demand in the angry face-fear situation condition.
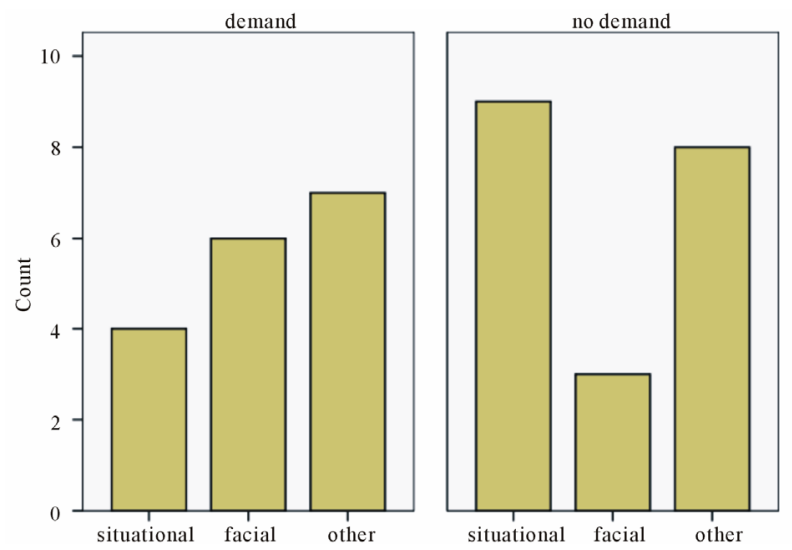

Figure 3.

Responses to facial, situational, and other information under low and high demand in the fear face-anger situation condition. 
sponse were grouped according to whether they corresponded to targeted facial information, situational information, or other undetermined information, responses corresponding to situational information were most frequent with low demand in the sad face-disgust situation conditions, $X^{2}(2)=12.4, \mathrm{p}<.01$, and the angry face-fear situation conditions, $X^{2}(2)=5.2$, $p<.07$. With high demand, responses corresponding to facial information were most frequent in the sad face-disgust situation condition, $X^{2}(2)=5.8, \mathrm{p}<.05$, and the angry face-fear situation conditions, $X^{2}(2)=5.1, \mathrm{p}<.08$.

Interestingly, responses corresponding to situational information were more frequent than those corresponding to facial information in the low demand fear face-anger situation, although the overall statistical test did not achieve significance, likely as a result of the high frequency of responses that did not correspond to either situational or facial information, $X^{2}(2)=$ $3.1, \mathrm{p}>.05$. Similarly, with high demand, responses corresponding to facial information were more frequent than those corresponding to situational information, albeit not significantly so, owing to the frequency of responses that did not correspond to either situational or facial information, $X^{2}(2)=.84, p>.05$. The data in Table 2 suggest that perceivers most often misidentified emotions in the fear face-anger situation as surprise.

Table 2.

The percentage of participants choosing each emotion in each experimental condition.

\begin{tabular}{|c|c|c|c|c|}
\hline \multirow{3}{*}{$\begin{array}{c}\text { Emotion } \\
\text { Happy }\end{array}$} & \multicolumn{2}{|c|}{ Low demand } & \multicolumn{2}{|c|}{ High demand } \\
\hline & \multicolumn{4}{|c|}{ Sad face—Disgust situation } \\
\hline & 0 & $(0)^{\mathrm{a}}$ & 0 & $(0)^{\mathrm{b}}$ \\
\hline Angry & 0 & $(0)$ & 0 & (0) \\
\hline Sad & 20 & (8) & 59 & (92) \\
\hline Surprise & 5 & (0) & 6 & (4) \\
\hline Fear & 5 & (4) & 6 & (4) \\
\hline Disgust & 70 & (88) & 29 & 0 \\
\hline \multicolumn{5}{|c|}{ Angry face —-Fear situation } \\
\hline Happy & 0 & (0) & 0 & (0) \\
\hline Angry & 40 & (4) & 59 & (44) \\
\hline Sad & 0 & (0) & 0 & (0) \\
\hline Surprise & 0 & (16) & 0 & (16) \\
\hline Fear & 50 & (76) & 18 & (12) \\
\hline Disgust & 10 & (4) & 23 & (28) \\
\hline \multicolumn{5}{|c|}{ Fear face—Anger situation } \\
\hline Нарру & 0 & (0) & 0 & (0) \\
\hline Angry & 45 & (76) & 24 & (4) \\
\hline Sad & 0 & (0) & 0 & (0) \\
\hline Surprise & 35 & (20) & 29 & (48) \\
\hline Fear & 15 & (4) & 35 & (44) \\
\hline Disgust & 5 & (0) & 12 & (4) \\
\hline
\end{tabular}

Note: The emotion selected most often italicized and appears in bold. ${ }^{\text {a Percent- }}$ ages in parentheses were reported by Carroll and Russell (1996) in their face plus situation $(\mathrm{F}+\mathrm{S})$ condition. ${ }^{\mathrm{b}}$ Percentages in parentheses were reported by Carroll and Russell (1996) in their face alone (F) condition.

\section{Discussion}

What do these findings reveal about emotion judgments and the debate surrounding the relative dominance of facial and situational information? First, they suggest that attention to facial and situational information is in part moderated by demands placed on perceivers' cognitive resources. When cognitive demands are high, perceivers rely more on facial information suggesting the primacy of facial cues in the emotion judgment process. When perceivers are not compelled to exert high cognitive effort, they make greater use of situational information suggesting its inferential, resource-demanding nature. A second, a broader implication of these findings is that mechanisms involved in processing nonverbal (emotional) cues coincide with general social-cognitive processes identified in person perception research.

In addition to the predicted effects, several additional noteworthy effects were observed. In particular, some emotions (e.g., surprise) were erroneously identified in high percentages despite the availability of explicit facial or situational information. Other researchers have found similar recognition errors (Carroll \& Russell, 1996; Ekman \& Friesen, 1975). Such errors point to unresolved questions about the nature of basic emotions and the cues that support their perception. It was also observed that some perceivers made emotion judgments that were not consistent with either the intended facial or situational information. These errors suggest that additional cognitive mechanisms may be invoked when perceivers are confronted with conflicting emotion information. For example, some perceivers may attempt to utilize integrative strategies such as the ones Asch (1946) suggested perceivers use when combining varied trait information about people into an overall impression. Determining what other cognitive mechanisms impact on emotion judgments and what factors guide their use are important questions to address.

A final issue to consider is the nature of facial versus situational information. In this and previous research, situational information was verbal in nature. Gilbert and Krull (1988) have argued that drawing inferences from nonverbal behaviors is more automatic than drawing inferences from verbal behavior. Thus, the effects observed here likely also involve reactions to specific types of stimulus information. In everyday contexts, situations embody a variety of verbal and nonverbal cues. Moreover, facial cues are embedded in situational contexts in complex and diverse ways. Knowing how to best distinguish and represent these different sources of information experimentally is a challenge researchers must confront.

\section{References}

Asch, S. E. (1946). Forming impressions of personality. Journal of Abnormal and Social Psychology, 41, 258-290. doi:10.1037/h0055756

Buck, R. (1984). The communication of emotion. New York: Guilford Press.

Carroll, J. M., \& Russell, J. A. (1996). Do facial expression signal specific emotions? Judging emotion from the face in context. Journal of Personality and Social Psychology, 70, 205-218. doi:10.1037/0022-3514.70.2.205

Darwin, C. (1872). The expression of the emotions in man and animals. London: J. Murray. doi:10.1037/10001-000

DePaulo, B., \& Friedman, H. S. (1998). Nonverbal communication. In D. T. Gilbert, S. T. Fiske, \& G. Lindzey (Eds.), The handbook of social psychology (Vol. 2, pp. 3-34). New York: McGraw-Hill.

Ekman, P. (1992). An argument for basic emotions. Cognition and 
Emotion, 6, 169-200. doi:10.1080/02699939208411068

Ekman, P., \& Friesen, W. V. (1975). Unmasking the face. Englewood Cliffs, NJ: Prentice-Hall.

Ekman, P., Friesen, W. V., \& Ellsworth, P. (1982). What are the relative contributions of facial behavior and contextual information to the judgment of emotion? In P. Ekman (Ed.), Emotion in the human face (pp. 111-127). Cambridge: Cambridge University Press.

Fernandez-Dols, J. M., Sierra, B., \& Ruiz-Belda, M. A. (1993). On the clarity of expressive and contextual information in the recognition of emotions: A methodological critique. European Journal of Social Psychology, 23, 195-202. doi:10.1002/ejsp.2420230207

Fernandez-Dols, J. M., Wallbott, H., \& Sanchez, F. (1991). Emotion category accessibility and the decoding of emotion from facial expression and context. Journal of Nonverbal Behavior, 15, 107-123. doi:10.1007/BF00998266

Fridlund, A. J. (1994). Human facial expression: An evolutionary view. San Diego: Academic Press.

Gilbert, D. T., \& Krull, D. S. (1988). Seeing less and knowing more: The benefits of perceptual ignorance. Journal of Personality and Social Psychology, 54, 193-201. doi:10.1037/0022-3514.54.2.193

Gilbert, D. T., Pelham, B. W., \& Krull, D. S. (1988). On cognitive busyness: When person perceivers meet persons perceived. Journal of Personality and Social Psychology, 54, 733-740. doi:10.1037/0022-3514.54.5.733

Goodenough, F. L., \& Tinker, M. A. (1931). The relative potency of facial expression and verbal description of stimulus in the judgment of emotion. Comparative Psychology, 12, 365-370. doi:10.1037/h0071381

Knapp, M. L., \& Hall, J. A. (2007). Nonverbal communication in human interaction (5th ed.) Wadsworth: Thomas Learning.

Izard, C. E. (1994). Innate and universal facial expressions: Evidence from developmental and cross-cultural research. Psychological Bulletin, 115, 288-299. doi:10.1037/0033-2909.115.2.288

Nakamura, M., Buck, R., \& Kenny, D. A. (1990). Relative contribution of expressive behavior and contextual information to the judgment of the emotional state of another. Journal of Personality and Social Psychology, 59, 1032-1103. doi:10.1037/0022-3514.59.5.1032

Matsumoto, D., Yoo, S., Hirayama, S., \& Petrova, G. (2005). Development and validation of a measure of display rule knowledge: The display rule assessment inventory. Emotion, 5, 23-40. doi:10.1037/1528-3542.5.1.23

Patterson, M. L., \& Stockbridge, E. (1998). Effects of cognitive demand and judgment accuracy on person perception accuracy. Journal of Nonverbal Behavior, 22, 253-264. doi:10.1023/A:1022996522793

Russell, J. (1997). Reading emotions from and into faces: Resurrecting dimensional-contextual perspective. In J. Russell, \& J. M. Fernandez-Dols (Eds.), The psychology of facial expression (pp. 295-320). Cambridge: Cambridge University Press.

Stanley, D. J., \& Meyer, J. P (2009). Two-dimensional affective space: A new approach to orienting the axes. Emotion, 9, 214-237.

Tomkins, S. S. (1962-1963). Affect, imagery, consciousness (Vol. 1-2). New York: Springer. doi:10.1037/a0014612 